\title{
A produção acadêmica realizada em língua portuguesa sobre Recursos Educacionais Abertos: Um estudo bibliométrico
}

\author{
Tel Amiel ${ }^{1}$, Airton Zancanaro ${ }^{2}$ \\ ${ }^{1}$ Núcleo de Informática Aplicada à Educação \\ Universidade de Campinas, SP - Brasil \\ ${ }^{2}$ Departamento de Contabilidade \\ Universidade Regional de Blumenau, SC - Brasil \\ tamiel@unicamp.br, airtonza@gmail.com
}

\begin{abstract}
There is an increasing interest in "openness" in education and in this, no field gathers as much interest as Open Educational Resources (OER). Still, we know very little of about the academic production regarding OER in Portuguese. Through a bibliometric study we identified works available in repositories, journals and proceedings as well as other non-indexed works produced by researchers in CPLP (community of Portuguese-speaking countries). A total of 355 locations were searched, and 107 works became the corpus of the analysis. Publications were analysed in regards to year of publication, where they were published, main institutions, and cited references. An analysis of this production makes it possible to expand the visibility of research on OER in Portuguese and can help promote the collaboration between authors who might discuss similar issues but are unaware of the research of others with similar interests.
\end{abstract}

Resumo. Há atualmente um interesse em investigar o conceito de "abertura" na educação e nenhum tema angaria tanto a atenção quanto Recursos Educacionais Abertos (REA). No entanto, pouco sabemos sobre o que é produzido no âmbito acadêmico em torno dos REA em língua portuguesa. Por meio de um estudo bibliométrico, buscamos identificar trabalhos publicados repositórios, periódicos e anais, bem como, aqueles que não indexados, produzidos por pesquisadores da Comunidade de Países de Língua Portuguesa (CPLP). Foram pesquisados 355 locais, formando um conjunto de 107 trabalhos. Foram analisadas as publicações realizadas por ano, os principais locais em que os trabalhos foram publicados, as principais instituições e referências citadas. A análise dessa produção possibilita aumentar a visibilidade da produção em torno de REA em língua portuguesa, e promover a colaboração entre autores que muitas vezes abordam a mesma temática, mas não tem conhecimento do trabalho de outrem.

\section{Introdução}

Há um renovado interesse na centralidade de "recursos" na educação, e isso se deve principalmente ao crescimento do acesso a Internet e da capacidade produtiva em torno de novas mídias (Hill \& Hannafin, 1997). Em alinhamento com essas mudanças 


\section{CBIE-LACLO 2015}

Anais dos Workshops do IV Congresso Brasileiro de Informática na Educação (CBIE 2015)

consolidou-se um movimento chamado Recursos Educacionais Abertos (REA) que hoje é o maior responsável (senão o mais exitoso) por um renovado interesse na abertura em educação. O movimento REA tem como objetivo fomentar a produção e disseminação de conteúdo educacional com liberdade de uso, reuso e adaptação.

De particular relevância para o conceito de "abertura" é sua articulação em diversas esferas de atuação, complementares a educação, como a ciência aberta, os dados abertos e o Free/Libre and Open Source Software (FLOSS), que fazem parte de uma área pulsante de ativismo nos espaços não-formais e que, cada vez mais, envolvem as instituições formais de ensino.

No Brasil, o trabalho em torno da temática REA tem, como em muitos outros países, crescido rapidamente. No entanto, pouco sabemos sobre o que é produzido no âmbito acadêmico em torno dos REA. Há crescente preocupação com dominação de países ricos e inglês-falantes na produção e disseminação de recursos abertos (Amiel, 2013). A mesma preocupação se aplica ao conhecimento disseminado sobre REA e Educação Aberta (EA), que por força das demandas acadêmicas, encontra pressão para que seja disseminado em língua inglesa e em periódicos indexados (Rego, 2014). Dado esse cenário, nos perguntamos, o que é produzido no âmbito acadêmico sobre REA em língua portuguesa? Trabalhos anteriores, como os de Arimoto e Barbosa (2012), Cobo (2013) e Zancanaro, Todesco e Ramos (2015), os autores fizeram mapeamentos da produção científica relacionadas aos REA, entretanto não foram identificadas pesquisas que abordassem especificamente trabalhos publicados em língua portuguesa.

Este estudo parte de um projeto que se iniciou em 2013 com o objetivo de catalogar a crescente produção acadêmica em língua portuguesa em torno dos REAs. Desde o primeiro uso do termo em um congresso na UNESCO em 2002, trabalhos em torno do conceito têm crescido sensivelmente. O projeto OER Knowledge Cloud (http://oerknowledgecloud.org), dedicado a catalogar publicações sobre REA em inglês apresenta hoje pouco mais de 1.000 itens. Buscamos com esse trabalho apontar, de maneira sistemática, os atores, produções e temáticas em torno de REA não somente no Brasil, mas ao redor do mundo, com enfoque na produção em língua portuguesa.

\section{Procedimentos metodológicos}

Uma pesquisa bibliométrica caracteriza-se pela análise da produção científica de modo a identificar indicadores que possam retratar o desenvolvimento de uma determinada área do conhecimento (Bufrem e Prates, 2005). Para tal, este estudo bibliométrico foi realizado em sete etapas que serão descritas na sequência.

\section{Etapa 1: Definição dos termos de busca}

A pesquisa bibliográfica teve como meta identificar artigos que abordem a temática Recursos Educacionais Abertos, que foram publicados em acesso aberto, no idioma português e com particular enfoque na área de Educação. Para isso foi utilizado o seguinte termo de busca: "recursos educacionais abertos" OU "recursos abertos" OU "recursos digitais abertos" OU "recurso educacional aberto" OU "recurso aberto" OU "objetos educacionais abertos" OU "objeto educacional aberto". A opção por estes termos se dá pelo fato que, muitas vezes, são utilizados como sinônimos para REA.

Etapa 2: Definição dos locais para a realização das buscas 


\section{CBIE-LACLO 2015}

Anais dos Workshops do IV Congresso Brasileiro de Informática na Educação (CBIE 2015)

A pesquisa deu-se em dois momentos, seguindo os critérios de seleção dos trabalhos descritos na Etapa 1. O primeiro momento foi iniciado em 2013 até maio de 2015 e teve como enfoque a seleção dos trabalhos depositados nos repositórios abertos e periódicos: Scielo, @Educa, Banco de teses/CAPES, Biblioteca Digital Brasileira de Computação e o Repositório Científico de Acesso Aberto de Portugal. Além disso, foram incluídos trabalhos publicados em anais de conferência que, sabidamente, trataram do assunto. Por último houve a inclusão de outros materiais identificados conforme a conveniência desta pesquisa e apontado em listas sobe o tema, como a REA Brasil.

No segundo momento, buscamos expandir a pesquisa para que o escopo incorporasse um maior número de produções em língua portuguesa desenvolvidas na Comunidade de Países de Língua Portuguesa (CPLP; Angola, Brasil, Cabo Verde, Guiné-Bissau, Guiné Equatorial, Moçambique, Portugal, São Tomé e Príncipe, e Timor Leste). Para tal, foram realizadas buscas em periódicos cadastrados no Directory of open Access Journals (DOAJ), classificadas pelo assunto Educação e em língua portuguesa. Também foram realizadas buscas nos repositórios dos países pertencentes a CPLP que estão cadastrados no Registry of Open Access Repositories (ROAR). Por fim, foram realizadas buscas no agregador internacional de bibliotecas WorldCat e no agregador de pesquisas científicas OpenAIRE. Vale destacar que estas buscas foram realizadas nos dias 16 e 17 de junho de 2015. Em ambos os momentos, nós focamos exclusivamente em publicações de acesso aberto, entendidas como aquelas onde os recursos estavam disponíveis abertamente para consulta e download.

\section{Etapa 3: Aplicação de critérios de seleção dos trabalhos}

Os termos de busca definidos na Etapa 1 foram utilizados para a realização de buscas nos repositórios, agregadores, periódicos e anais, definidos na Etapa 2. Os trabalhos identificados nas buscas, inicialmente foram lidos o título, o resumo e as palavras-chave e, em caso de dúvidas, houve a leitura do texto completo, como o propósito de retirar aqueles trabalhos que estavam fora do contexto de estudo. Além disso, foram selecionados trabalhos que não estavam indexados nos repositórios e periódicos. Neste caso, buscamos por livros, capítulos de livros, reportagens em revistas e relatórios técnicos que estivesse dentro do contexto do estudo.

Etapa 4: Inclusão dos trabalhos selecionados no software de gerenciamento de referencias bibliográficas

Após a seleção dos trabalhos relevantes ao contexto do estudo, aqueles trabalhos que não estavam registrados na base do software de gerenciamento foram inseridas manualmente. Para tanto utilizamos um grupo no Zotero (http://zotero.org), um sistema para gerenciamento de referências bibliográficas colaborativo e aberto, baseado em software livre. Isto permitiu organizar e formar um único conjunto de trabalhos contendo, além dos metadados, o link para o texto completo.

Etapa 5: Padronização e inclusão de informações além daquelas disponíveis nos metadados

Utilizando os metadados disponíveis no Zotero, criou-se uma nova base de dados para ampliarmos a análise das informações. Os metadados que estavam no gerenciador de referências bibliográficas, foram adicionados e padronizados manualmente. Incluímos também informações referentes ao vínculo institucional, ao georeferenciamento das 
CBIE-LACLO 2015

Anais dos Workshops do IV Congresso Brasileiro de Informática na Educação (CBIE 2015)

instituições em que os autores estão afiliados e as referências utilizadas pelos autores para compor os trabalhos.

Etapa 6: Análise dos dados

Tendo o conjunto de trabalhos selecionados e padronizados, foi possível gerar consultas e imagens que melhor interpretam os dados, alinhado aos objetivos da pesquisa.

\section{Resultados}

Nesta seção, são apresentados os resultados das análises e sínteses das informações selecionados a partir das buscas realizadas nas bases de dados, periódicos, anais de eventos e repositórios, bem como daqueles que foram identificados isoladamente.

\subsection{Resultados gerais da pesquisa}

Os dados da Tabela 1 referem-se ao resumo que reúne todos os dados coletados. Organizamos os resultados com base na origem dos trabalhos focando em repositórios, periódicos específicos, anais de eventos, agregadores de conteúdo e documentos avulsos, conforme explanado anteriormente.

Tabela 1. Resumo dos dados coletados nos momento 1 e 2

\begin{tabular}{|l|c|c|}
\hline & Total de locais pesquisados & Trabalhos identificados \\
\hline Repositórios & 216 & 48 \\
\hline Periódicos & 135 & 18 \\
\hline Agregadores & 2 & 4 \\
\hline Anais & 2 & 3 \\
\hline Avulsos & - & 34 \\
\hline Totais & 355 & 107 \\
\hline
\end{tabular}

Com base nas 107 publicações selecionadas podemos começar a mapear a produção acadêmica em torno de REA em língua portuguesa. No que tange o tipo de produção, 33 foram artigos publicados em periódicos e 29 em anais de eventos, 16 capítulos de livros e dois livros. No que tange a produção em nível de pós-graduação, encontramos 17 dissertações, quatro teses e um relatório de pós-doutorado. Ademais, identificamos dois relatórios técnicos, um trabalho de conclusão de curso, um artigo de revista e uma declaração. Na Tabela 2 apresenta os dados bibliográficos da pesquisa.

Tabela 2. Dados bibliográficos com base nas publicações selecionadas

\begin{tabular}{|l|c|}
\hline Dados bibliográficos & Frequência \\
\hline Diferentes fontes* nas quais os trabalhos selecionados foram publicados & 64 \\
\hline Autores únicos & 169 \\
\hline Instituições dos autores & 55 \\
\hline
\end{tabular}


CBIE-LACLO 2015

Anais dos Workshops do IV Congresso Brasileiro de Informática na Educação (CBIE 2015)

\begin{tabular}{|l|c|}
\hline Países das instituições dos autores & 6 \\
\hline Palavras-chave distintas utilizadas & 206 \\
\hline Referencias distintas utilizadas & 3.242 \\
\hline
\end{tabular}

* Fontes, neste caso, refere-se ao local onde o trabalho foi publicado. Em pesquisas acadêmicas, consideramos como fonte as instituições que as publicaram.

\subsection{Tendências temporais}

Ao analisar os 107 trabalhos, observa-se que as primeiras publicações aconteceram em 2007, cinco anos após o primeiro uso do termo "Recursos Educacionais Abertos" em um seminário da UNESCO (2002). Nesse ano ocorreram duas publicações. Primeiro, a Declaração da Cidade do Cabo (2007), originária de um evento promovido pela Open Society Institute e da Fundação Shuttleworth na Africa do Sul que teve como propósito promover os recursos abertos (originalmente escrito em inglês e depois traduzido para o Português). Segundo, um artigo escrito por Dutra e Tarouco (2007) intitulado "Recursos Educacionais Abertos (Open Educational Resources)" que buscou fazer um apanhado geral sobre o tema, partindo da recente experiência em torno do OpenCourseWare (MIT). A Tabela 3 apresenta a evolução das publicações sobre REA em português.

Tabela 3. Publicações por ano

\begin{tabular}{|l|l|l|l|l|l|l|l|l|l|c|}
\hline Documento & 2007 & 2008 & 2009 & 2010 & 2011 & 2012 & 2013 & 2014 & 2015 & Total \\
\hline Artigo de revista & & & & & & & & 1 & & 1 \\
\hline Capítulo de livro & & & & & & 15 & & 1 & & 16 \\
\hline Anais de eventos & & 1 & & 1 & 1 & 9 & 9 & 8 & & 29 \\
\hline Dissertação & 1 & & 1 & 2 & 2 & & 5 & 5 & 2 & 17 \\
\hline Declaração & 1 & 1 & 2 & 1 & 2 & 5 & 8 & 10 & 3 & 33 \\
\hline $\begin{array}{l}\text { Artigos de } \\
\text { periódicos }\end{array}$ & & & & & 1 & 1 & & & & 2 \\
\hline Livro & 2 & 2 & 4 & 4 & 6 & 33 & 24 & 26 & 6 & 107 \\
\hline $\begin{array}{l}\text { Relatório de Pós- } \\
\text { Doutorado }\end{array}$ & & & & & & & 1 & 1 & & 2 \\
\hline Relatório técnico & & & & & 3 & & & 1 & 4 \\
\hline Tese & & & & & & & & 1 \\
\hline $\begin{array}{l}\text { Trabalho de } \\
\text { conclusão de curso }\end{array}$ & & & & & & & & & & 1 \\
\hline Total
\end{tabular}

Em 2012 houve um aumento considerável nas publicações sobre REA (33 publicações). Isto se deve ao lançamento do livro "Recursos Educacionais Abertos: 


\section{CBIE-LACLO 2015}

Anais dos Workshops do IV Congresso Brasileiro de Informática na Educação (CBIE 2015)

Práticas colaborativas e políticas públicas" conhecido como "Livro REA" (http://livrorea.net.br; 11 trabalhos) e a publicação de "Recursos Educacionais Abertos e Redes Sociais: coaprendizagem e desenvolvimento profissional" (quatro trabalhos). Além disso, neste ano, houve a promoção do "I Workshop Recursos Educacionais Abertos" (I WREA) como parte do Congresso Brasileiro de Informática na Educação (CBIE), no qual oito artigos foram publicados seguindo essa temática. Neste mesmo ano, contabilizamos cinco publicações em periódicos científicos, três teses acadêmicas e um livro. Este ano também coincide com o Congresso Global sobre REA realizado na UNESCO em Paris que originou uma "declaração" global para o avanço de REA enquanto política pública e práticas institucionais (Declaração REA de Paris, 2012). Vemos, portanto, 2012 como um ano de "inflexão" em torno da produção sobre a temática.

Outras conferências foram realizadas: seis em 2013 e sete em 2014 em que trabalhos que tratam da temática REA foram aceitos. Destaca-se o "III Colóquio LusoBrasileiro de Educação a Distância e E-learning" realizado em 2013 na qual foram publicados quatro trabalhos e "III Congresso Brasileiro de Informática na Educação" realizado em 2014 em que foram publicados dois artigos sobre a temática tratada neste trabalho.

Nota-se que as publicações em periódicos científicos vêm aumentando com o passar do tempo: houveram cinco em 2012, oito em 2013, dez em 2014 e, por hora, três em 2015. A publicação de artigos acadêmicos pode sugerir uma maior maturidade na produção acadêmica, visto que usualmente são produtos de pesquisas e reflexões mais intensas sobre o tema.

\subsection{Principais fontes de publicações}

Os 107 trabalhos selecionados foram originários de 64 diferentes fontes, sendo três principais: o Livro REA (11), o I Workshop REA (8) e a Revista RENOTE (7). O primeiro, organizado por Santana, Rossini e Pretto foi produto de um edital lançado pelo Comitê Gestor da Internet (CGI). É uma compilação de reflexões teóricas, experiências e depoimentos, além de cinco entrevistas sobre a temática REA (não incluídas como produção nos termos desse trabalho).

Já o segundo, foi produto de um evento integrado ao Congresso Brasileiro de Informática na Educação (CBIE). O evento foi organizado pelo Grupo de Trabalho Educação Aberta (UNICAMP), como seguimento de um evento internacional realizado no ano anterior (I Symposium on OER: Issues for Localization and Globalization). Ambos são, portanto, produtos de situações que podem ser consideradas excepcionais dentro do fluxo de produção acadêmica (artigos, dissertações e teses). A última é uma revista científica mantida pela UFRGS, com o propósito de publicar e divulgar trabalhos na área de informática na educação.

\subsection{Principais instituições e países}

As publicações analisadas foram escritas por 169 diferentes autores que estão afiliados a 55 diferentes instituições. A Tabela 4 apresenta as principais instituições, destacando que o Brasil possui 77,5\% do total de autores, Portugal tem 14,2\%, Reino Unido tem $5,9 \%$, Estados Unidos tem 1,2\% e Alemanha e África do Sul com 0,6\% completam a 
CBIE-LACLO 2015

Anais dos Workshops do IV Congresso Brasileiro de Informática na Educação (CBIE 2015)

lista de países que possuem autores publicando em língua portuguesa sobre a temática REA.

Tabela 4. Instituições com maior número de autores afiliados

\begin{tabular}{|c|c|c|c|c|}
\hline Instituição & $\begin{array}{l}\text { Total de autores } \\
\quad \text { afiliados }\end{array}$ & $\%$ & Cidade & País \\
\hline $\begin{array}{l}\text { Universidade Federal do Rio Grande } \\
\text { do Sul }\end{array}$ & 13 & 7,7 & Porto Alegre & Brasil \\
\hline Universidade Aberta - Portugal & 12 & 7,1 & Lisboa & Portugal \\
\hline Open University & 10 & 5,9 & Milton Keynes & Reino Unido \\
\hline $\begin{array}{l}\text { Universidade Federal de Santa } \\
\text { Catarina }\end{array}$ & 10 & 5,9 & Florianópolis & \multirow[t]{5}{*}{ Brasil } \\
\hline Universidade Federal de Santa Maria & 10 & 5,9 & Santa Maria & \\
\hline USP & 10 & 5,9 & São Paulo & \\
\hline Universidade Federal de São Carlos & 6 & 3,6 & São Carlos & \\
\hline Universidade Federal do Ceará & 6 & 3,6 & Fortaleza & \\
\hline
\end{tabular}

A partir dos dados das instituições em que os autores estão afiliados é possível gerar um mapa (Figura 1) demonstrando a localização geográfica dos pesquisadores sobre REA. Os círculos em vermelho representam os pontos em que possuem número de pesquisadores trabalhando no tema REA. Quanto maior o círculo, maior é a quantidade de autores.

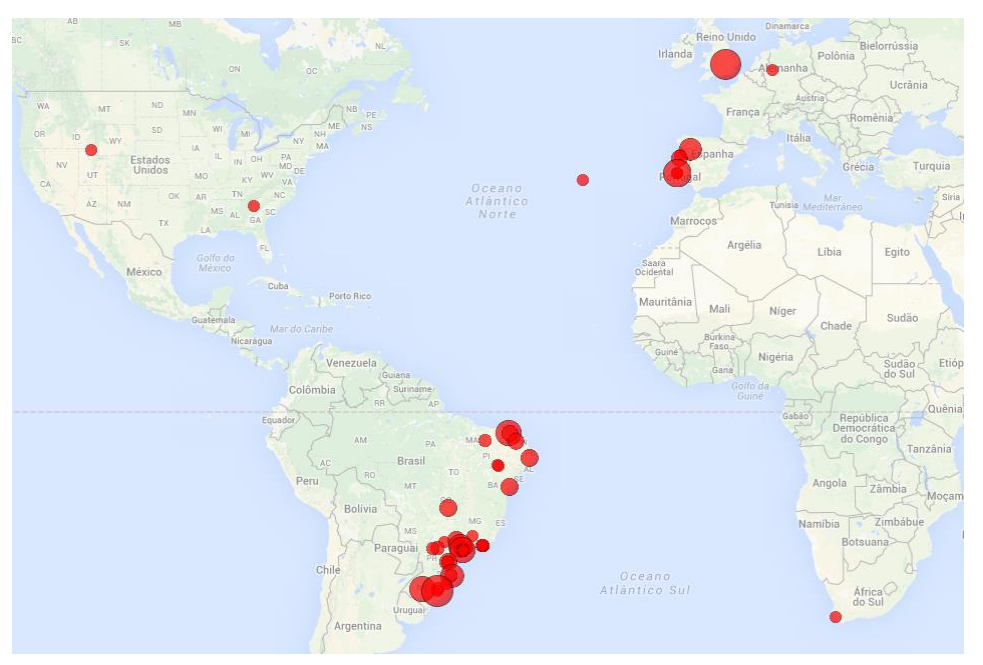

Figura 1. Mapa da localização dos pesquisadores

Podemos notar que dos 131 pesquisadores afiliados a instituições brasileiras, os estados que possuem maior concentração de pesquisadores são: São Paulo (41), Rio Grande do Sul (26), Paraná (14), Santa Catarina (13) e Ceará com 11. Em São Paulo, as instituições com maior número de autores são: USP com 10, Universidade Federal de 
São Carlos com seis e UNICAMP e PUC-SP com cinco. Nos outros estados (RS, PR, $\mathrm{SC}$ e $\mathrm{CE}$ ) as pesquisas se concentram principalmente nas Universidades Federais. Em Portugal há uma concentração de pesquisadores principalmente na Universidade Aberta de Portugal, no Instituto Politécnico de Bragança e na Universidade de Aveiro. Já no Reino Unido todos os pesquisadores estão vinculados a Open University. É importante notar a limitada presença de outros países cuja língua oficial é o português.

\subsection{Principais referências citadas}

Com base nos 107 trabalhos analisados, foi possível identificar que os autores utilizaram 3.242 diferentes referências bibliográficas para compor os seus trabalhos. Os trabalhos mais citados estão descritos na Tabela 5.

Tabela 5. Principais referências utilizadas

\begin{tabular}{|l|c|l|c|}
\hline Autor(es) & $\begin{array}{c}\text { Ano da } \\
\text { publicação }\end{array}$ & Título & $\begin{array}{c}\text { Número de } \\
\text { citações }\end{array}$ \\
\hline Jan Hylén & 2006 & $\begin{array}{l}\text { Open Educational Resources: } \\
\text { Opportunities and Challenges }\end{array}$ & 18 \\
\hline Neil Butcher & 2011 & $\begin{array}{l}\text { A Basic Guide to Open Educational } \\
\text { Resources (OER) }\end{array}$ & 15 \\
\hline $\begin{array}{l}\text { Bianca Santana, } \\
\text { Carolina Rossini e } \\
\text { Nelson De Luca Pretto }\end{array}$ & 2012 & $\begin{array}{l}\text { Recursos Educacionais Abertos: } \\
\text { práticas colaborativas e políticas } \\
\text { públicas }\end{array}$ & 13 \\
\hline UNESCO & 2002 & $\begin{array}{l}\text { Forum on the Impact of Open } \\
\text { Courseware for Higher Education in } \\
\text { Developing Countries }\end{array}$ & \\
\hline Stephen Downes & 2007 & $\begin{array}{l}\text { Models for Sustainable Open } \\
\text { Educational Resources }\end{array}$ & \\
\hline
\end{tabular}

Os três trabalhos mais citados na língua inglesa são referências gerais sobre a temática. O trabalho de Hylén (2006) é produto de um relatório da OECD que faz um apanhado geral sobre REA: conceitualiza a temática, esclarece questões que fazem com que as instituições estejam engajadas na disponibilização de materiais abertos e apresenta os desafios para o crescimento do movimento. Já o trabalho de Butcher (2011) foi traduzido para o português pelo CETIC.br. Nele, o autor explora o conceito de REA e aponta argumentações em favor do movimento, incluindo questões políticas e licenças de uso. O Forum on the Impact of Open Courseware for Higher Education in Developing Countries foi um evento realizado em Paris, em julho de 2002, pela UNESCO, em colaboração com a William and Flora Hewlett Foundation e a Western Cooperative for Educational Telecommunications (WCET). É uma referência utilizada para denotar o primeiro uso formal do termo Open Educational Resources (OER; Recursos Educacionais Abertos), como uma alternativa a outros já existentes.

O Livro REA (Santana, Rossini e Pretto, 2012) foi uma realização da Casa da Cultura Digital e da Edufba com financiamento do CGI.br, da Open Society Foundation e apoiado pela Comunidade REA-Brasil. Teve como proposta uma visão ampla do que sejam os REAs, apontando questões, dilemas e iniciativas com enfoque no Brasil. Deste 


\section{CBIE-LACLO 2015}

Anais dos Workshops do IV Congresso Brasileiro de Informática na Educação (CBIE 2015)

livro, o capítulo "Educação aberta: Configurando ambientes, práticas e recursos educacionais", de Tel Amiel, faz um apanhado teórico sobre educação aberta, trazendo reflexões sobre os recursos e as práticas abertas. Outros artigos com alto número de referências incluem "Professores-autores em rede" de Nelson Pretto com 11 referências; "REA: O debate em política pública e as oportunidades para o mercado" de Carolina Rossini com 10 referências; e "Educação Aberta: histórico, práticas e o contexto dos recursos educacionais abertos" de Andreia Inamorato dos Santos com seis referências. Por último, Downes (2007) aborda a importância dos REA, conceitualiza os termos recursos e abertos, esclarece a sustentabilidade os diferentes modelos de negócios que podem auxiliar na sustentabilidade de projetos de REA.

\section{Considerações finais}

Através da aplicação de técnicas bibliométricas, este artigo procurou identificar as características das pesquisas realizadas sobre REA publicados em língua portuguesa. Os dados utilizados nesta pesquisa foram recuperados depois de buscas em 355 locais e a seleção daqueles materiais que estavam no contexto do estudo. Apontamos abaixo algumas considerações técnicas e limitações do estudo.

A grande quantidade de locais pesquisados revela a falta de agregadores que forneçam resultados confiáveis nas buscas. Tanto o site do ROAR quando do DOAJ possuem uma opção global para busca, no entanto, os resultados são diferentes quando realizados separadamente em cada site do periódico/repositório. Para resolver esse problema efetuamos a busca individualmente em cada local especificado, o que demandou um tempo maior de consulta. Além disso, destacamos que em 36,5\% dos trabalhos analisados os autores não definiram as palavras-chave. Isto impediu uma maior precisão das análises neste quesito, além de dificultar a localização dos trabalhos pelos motores de buscas.

Destacamos que pouco menos de um terço $(31,8 \%)$ dos trabalhos não estavam indexados em sites de revistas ou repositórios, sendo incluídos de forma avulsa no conjunto final de artigos. Essa inclusão se fez necessária devido à natureza da produção em torno da temática, o que julgamos ser mais realista do que uma busca imparcial somente em periódicos indexados, o que constituiria um rigor sem validade. Nos parece certo que com o crescimento da produtividade sobre o tema nas vias tradicionais (como pós-graduação), haverá um crescimento da produção nos meios que são valorizados formalmente pela academia (periódicos indexados).

Um apontamento correlato é que, dada a ênfase crescente em torno da publicação de artigos em periódicos internacionais (particularmente em língua inglesa), a produção acadêmica em países de língua portuguesa é sub-representada. Futuros estudos podem investigar as redes de produção em torno de REA e EA partindo da localização dos autores independente do idioma da produção. Isso poderá, por exemplo, nos apontar à produção oriunda de outros países (que integram a CPLP) que se mostraram inexistentes nesse levantamento.

Por último, reconhecemos a limitação da imagem que representa a localização das instituições dos pesquisadores no formato de um artigo acadêmico. Essa figura juntamente com demais dados da pesquisa serão posteriormente publicados no site do grupo de trabalho. A análise da produção em torno de REA nos auxilia a mapear e 


\section{CBIE-LACLO 2015}

Anais dos Workshops do IV Congresso Brasileiro de Informática na Educação (CBIE 2015)

identificar atores e temáticas relevantes. Seguindo a meta da proposta inicial deste trabalho, buscamos com isso aumentar a visibilidade da produção em torno de REA em língua portuguesa, fomentar a integração entre os que publicam em português e promover a colaboração entre autores que muitas vezes abordam a mesma temática, mas que não tem conhecimento do trabalho de outrem. Esperamos que esse trabalho seja mais um passo a contribuir com esse objetivo.

\section{Referencias bibliográficas}

Amiel, T. (2013) Identifying Barriers to the Remix of Translated Open Educational

Resources. International Review of Research in Open and Distance Learning, 14(3), 126-144.

Arimoto, M. M., \& Barbosa, E. F. (2012). A systematic review of methods for developing open educational resources. Paper presented at the 20th International Conference on Computers in Education, ICCE 2012, Singapore.

Bufrem, L., \& Prates, Y. (2005). O saber científico registrado e as práticas de mensuração da informação. Ci. Inf., 34(2), 9-25.

Butcher, N. (2011). A Basic Guide to Open Educational Resources (OER). Paris, França: Unesco/Commonwealth of Learning.

Cobo, C. (2013). Exploration of Open Educational Resources in Non-English Speaking Communities. International Review of Research in Open and Distance Learning, 14(2), 106-128.

Declaração da Cidade do Cabo. (2007). Declaração de cidade do cabo para educação aberta: Abrindo a promessa de recursos educativos abertos. Disponível em: http://capetowndeclaration.org/

Downes, S. (2007). Models for sustainable open educational resources. Interdisciplinary Journal of Knowledge and Learning Objects, 3, 29-44.

Dutra, R. L. d. S., \& Tarouco, L. M. R. (2007). Recursos Educacionais Abertos (Open Educational Resources). RENOTE, 5(1).

Hill, J. R., \& Hannafin, M. J. (2001). Teaching and Learning in Digital Environments: The Resurgence of Resource-Based Learning. Educational Technology Research and Development, 49(3), 37-52.

Hylén, J. (2006). Open Educational Resources: Opportunities and Challenges (pp. 1-10). Paris: OECD.

Rego, T. C. (2014). Produtivismo, pesquisa e comunicação científica: entre o veneno e o remédio. Educ. Pesqui., 40(2), 325-346.

Santana, B., Rossini, C., \& Pretto, N. D. L. (2012). Recursos Educacionais Abertos: práticas colaborativas e políticas públicas. Salvador-BA; São Paulo-SP: Edufba/Casa da Cultura Digital.

UNESCO (2002) Forum on the Impact of Open Courseware for Higher Education in Developing Countries. Paris/França: UNESCO, 2002. 1-30.

UNESCO (2012). Declaração REA de Paris. Disponível em: $<$ http://www.unesco.org/new/en/communication-and-information/access-toknowledge/open-educational-resources/what-is-the-paris-oer-declaration>. Acesso em 20 Jul 2015.

Zancanaro, A., Todesco, J. L., \& Ramos, F. (2015). A bibliometric Mapping of Open Educational Resources. International Review of Research in Open and Distributed Learning, 16(1), 1-23. 